\title{
Supercritical angle Raman microscopy: a surface- sensitive nanoscale technique without field enhancement
}

\author{
Diana Serrano and Stefan Seeger
}

Raman scattering microscopy is a versatile tool for label-free imaging and molecular fingerprint analysis. Here, we provide the first demonstration that the selective collection of scattered signals exceeding the critical angle for total internal reflection enables surface-confined spontaneous Raman investigations at nanometre resolution. This high-axial selectivity leads to improved signal-to-background ratios, thus making this technique an excellent probe for surface-related molecular specimens. The richness of the spectroscopic information obtained through the supercritical angle Raman (SAR) collection path was proven by comparing its output with that of a parallel far-field collection path. Furthermore, we demonstrated that the proposed SAR technique is a versatile microscopy approach that can be used alone or in combination with amplified Raman modalities such as surfaceenhanced resonance Raman scattering.

Light: Science \& Applications (2017) 6, e17066; doi:10.1038/lsa.2017.66; published online 20 October 2017

Keywords: near-field optics; Raman scattering; supercritical microscopy; surface science

\section{INTRODUCTION}

Raman scattering is a straightforward spectroscopic method of characterising complex molecular systems. It gathers rich chemical, structural and functional information, providing insight into central issues in chemical physics ${ }^{1}$, biophysics ${ }^{2}$ and materials science ${ }^{3}$. One shortcoming is that the typically low Raman scattering cross sections, in the range of $10^{-29}$ to $10^{-31} \mathrm{~cm}^{2}$, tend to result in weak signal levels ${ }^{4}$. Nevertheless, advances in the field of optical microscopy currently allow for the exploitation of spontaneous Raman scattering for label-free investigations of biological macromolecules, single cells and tissues ${ }^{5}$. The resolution of classical confocal Raman microscopy is limited by diffraction, and the signal-to-noise and signal-tobackground ratios remain challenging issues for the large-scale application of this technique $e^{6,7}$. In this context, alternative Raman modalities have emerged in recent years. These techniques can successfully achieve several-fold amplification of the Raman scattered signals. Among them, two main approaches can be distinguished: nonlinear or coherent Raman spectroscopy $(\mathrm{CRS})^{8}$, which takes advantage of nonlinear excitation schemes, and surface-plasmonrelated methods, such as the extensively used surface-enhanced Raman scattering $(\text { SERS })^{9}$. Furthermore, an interesting implementation of both approaches, namely, surface-enhanced coherent anti-Stokes Raman microscopy, has recently been reported ${ }^{10}$. This method allows for surface-sensitive super-resolution CRS to be performed by using an optical microscope built on the basis of the Kretschmann configuration $^{11}$.
Despite the prominent scientific output of amplified or enhanced Raman modalities ${ }^{10-17}$, the development of spontaneous Raman methods for nanoscale detection remains a subject of interest. Surface-enhanced Raman techniques also present disadvantages. The complex electromagnetic enhancement mechanism underlying SERS often leads to spectrally modified molecular fingerprints. The spectral content often slightly varies among substrates ${ }^{18}$ and even within the same substrate, depending on the SERS hotspot microenvironment ${ }^{19}$. In addition, the necessary proximity of metallic structures is likely to interfere in many chemical and biological processes ${ }^{20}$. However, coherent Raman scattering has been demonstrated to be very efficient for dynamic imaging ${ }^{21,22}$, although it does not allow for investigation of broad spectroscopic signatures, and it often requires complex optical pumping schemes with high-peak-power laser pulses ${ }^{23}$. Such pulses risk damaging the sample under investigation, a particularly critical consideration in the case of biologically active specimens ${ }^{24}$. In contrast, spontaneous Raman spectroscopy remains a versatile and minimally invasive Raman modality that allows complete, consistent and reproducible spectroscopic information to be obtained from a large variety of systems.

Among the very few techniques that have enabled superresolution spontaneous Raman investigations to date, approaches such as structure line illumination (SLI) Raman microscopy ${ }^{25}$ and total internal reflection (TIR) Raman microscopy ${ }^{26,27}$ have shown promising results. The technique presented in the current work, referred to as supercritical angle Raman (SAR) microscopy, is a powerful near-field-based method of conducting surface-sensitive 
non-amplified Raman scattering investigations. SAR microscopy takes advantage of the fact that dipole emitters embedded in a medium of refractive index $n_{1}$, and in proximity to a second medium of refractive index $n_{2}>n_{1}$ emit a non-negligible portion of their total radiated power into angles exceeding the critical angle for total internal reflection ${ }^{28,29}$. This supercritical radiation, also referred to as 'forbidden light', is due to the electromagnetic coupling of an emitter's near field with the high-refractive-index medium $^{28-30}$. Therefore, it is exponentially dependent on the distance from the emitter to the refractive index discontinuity. Thus, the selective collection of this forbidden light provides strong axial confinement to the refractive index discontinuity and, consequently allows for direct insight into surface-bound specimens and boundaries. The principle of supercritical angle detection has been successfully applied to fluorescent probes, thereby giving rise to so-called supercritical angle fluorescence (SAF) microscopy ${ }^{31}$. It has also been exploited in nanoscopy techniques derived from SAF, such as direct optical nanoscopy with axially localised detection (DONALD) ${ }^{32}$. Nevertheless, prior to this study, it had never been applied to Raman scattering collection. The extension of the principle from fluorescence to label-free Raman scattering is an important step forward in the field of high-resolution microscopy and surface science. The intrinsic structural and functional spectroscopic information contained in the Raman spectra, which is not accessible through dye-labelled fluorescence investigations, is crucial for gaining an understanding of complex surface phenomena, such as molecular aggregation ${ }^{33}$ or surface functionalization $^{34}$.

In this work, we provide the first demonstration that Raman scattering beyond the critical angle for total internal reflection can be efficiently collected, and we present the advantages of this microscopy technique for probing sub-micrometre volumes and nanoscale specimens without a need for field amplification or signal enhancement.

\section{MATERIALS AND METHODS}

Supercritical angle near-field and classical undercritical angle far-field Raman scattered signals, hereafter referred to as 'supercritical angle' Raman (SAR) signals and 'undercritical angle' Raman (UAR) signals in reference to their fluorescence analogues ${ }^{35}$, were simultaneously collected by using a custom-built objective consisting of a parabolic mirror and a multilens system with a numerical aperture equal to 1 . The parabolic element collected signals at angles between $60^{\circ}$ and $80^{\circ}$. Thus, this method gathers essentially all SAR scattering for a water/ glass interface characterised by a critical angle of $61.1^{\circ}$. Meanwhile, the inner optics focus the excitation and collect UAR scattering between $0^{\circ}$ and $41^{\circ}$. The custom-built objective was incorporated into an optical microscopy set-up prepared for imaging and spectral analysis (Figure 1). A power-tuneable diode laser at $633 \mathrm{~nm}$ applied the excitation. After the objective, the collimated UAR and SAR beams appeared transversally as a $5-\mathrm{mm}$ diameter circle surrounded by a 6-8 mm collimated ring, respectively. These two concentric beams were separated by a $45^{\circ}$ mirror mounted on a glass window before detection. As displayed in Figure 1, two different detection paths were available for the separated beams. Thus, the separated beams could be focused into two identical single-photon avalanche diodes (SPADs) with an active area of $180 \mu \mathrm{m}$ or could be injected through two identical fibre couplers into a $200 \mu \mathrm{m}$ core multimode fibre. The fibre output was connected to a spectrometer equipped with a low-noise CCD camera cooled to $-70^{\circ} \mathrm{C}$. The detection paths were switched by means of flip mirrors. Initial suppression of the Rayleigh scattering was performed with a dichroic beam splitter and several long-pass spectral filters along the collection paths. Furthermore, a band-pass filter was used in front of the SPADs to select the $1000 \mathrm{~cm}^{-1}$ peak in the polystyrene experiment. Images were recorded by using a customwritten Borland C++ program, whereas LabSpec (Horiba, Irvine, CA, USA) was used for spectral acquisitions. The images and spectra were then processed in MATLAB. A constant background was subtracted from both the UAR and SAR spectra. For clarity, the SAR spectra were

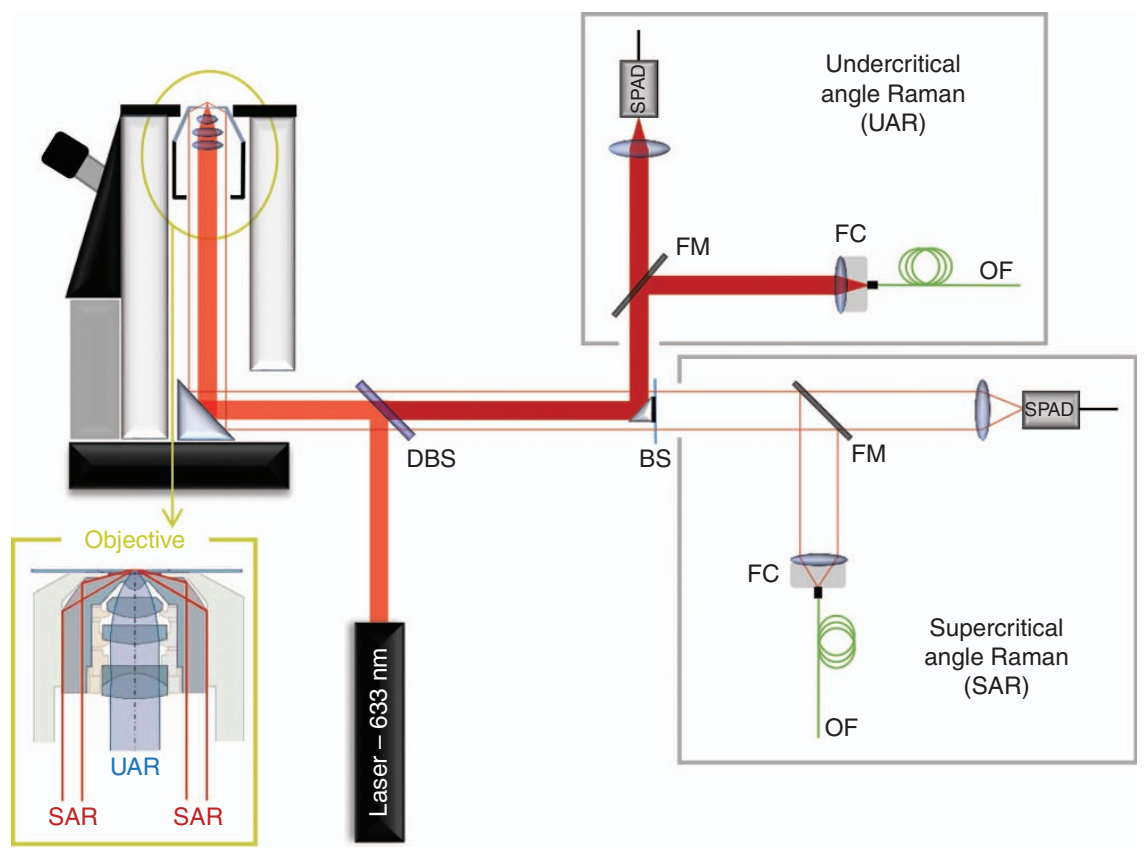

Figure 1 Experimental set-up scheme. DBS, dichroic beam splitter; FM, flip mirror; BS, beam splitter, referring to the $45^{\circ}$ split mirror; FC, fibre coupler; OF, optical fibre; and SPAD, single-photon avalanche diode. The detail view of the objective shows the multilens focusing and far-field collection system as well as the parabolic element for supercritical angle scattering collection. 

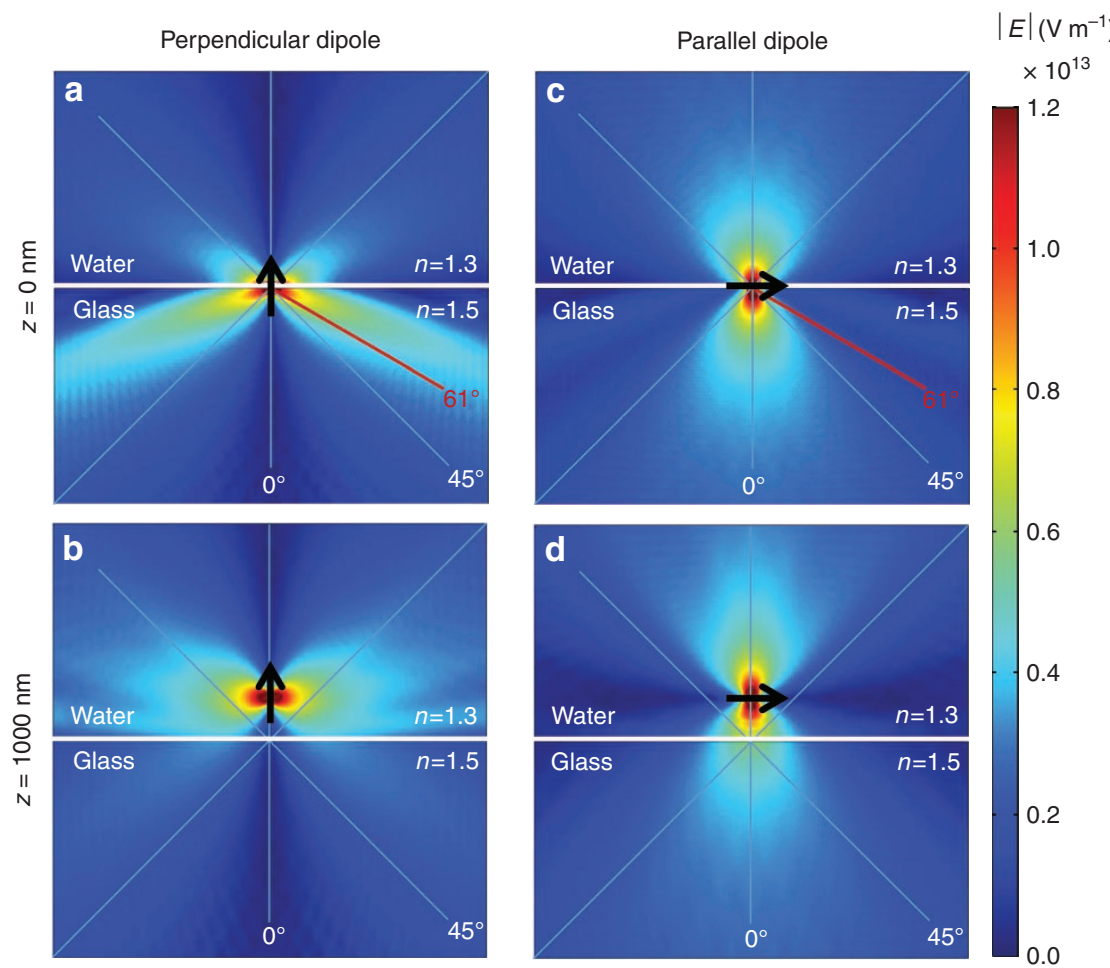

Figure 2 Angular distributions of the radiation emitted by a punctual incoherent dipole located at a distance ' $z$ ' from a water/glass interface $\left(\theta_{c}=61.1^{\circ}\right)$. (a) Dipole oriented perpendicular to the interface at $z=0$. In this case, a large amount of the radiated power is emitted at high angles towards the lower halfspace (glass). (b) For $z=1000 \mathrm{~nm}(z>\lambda=632 \mathrm{~nm})$, almost all of the emission occurs towards the upper half-space (water), because of total internal reflection. (c) Dipole oriented parallel to the interface at $z=0$ and (d) at $z=1000 \mathrm{~nm}$. Radiation patterns from ensembles of randomly oriented dipoles were computed by adding the patterns of parallel and perpendicular dipoles 29,30 .

also smoothed by means of adjacent averaging with a 5-point moving window.

\section{RESULTS AND DISCUSSION}

The angular radiation patterns of a dipole emitter close to a water $\left(n_{1}=1.333\right)$ /glass $\left(n_{2}=1.523\right)$ interface for various dipole orientations and various distances from the refractive index discontinuity are displayed in Figure 2. In the simulation, the extinction of the supercritical radiation with increasing axial distance ' $z$ ' can be clearly observed (Figure $2 \mathrm{a}$ and $2 \mathrm{~b}$ ). This extinction was not observed for the classical undercritical radiation patterns (Figure $2 \mathrm{c}$ and $2 \mathrm{~d}$ ), which propagated towards the lower half-space (glass) regardless of the distance from the emitter to the refractive index interface (see the Supplementary Information for detailed simulations). The orientation of the induced dipoles determined the proportion of the radiated power emitted as supercritical and undercritical.

Parallel SAR and UAR imaging of an aqueous suspension of polystyrene microspheres demonstrates the surface selectivity of SAR detection. In contrast to the UAR signal (Figure 3a), the SAR signal was sensitive to only microspheres on the coverslip surface, offering very good surface contrast and complete rejection of scattering in higher planes (Figure $3 \mathrm{~b}$ ). To obtain further insight into the SAR axial confinement and the consequent axial super-resolution, we measured the evolution of the SAR and UAR intensities along the radius of a single polymer microsphere. After a stable microsphere on the coverslip surface was identified (Figure 3c), the microscope objective was placed at its centre, $X_{0}$, and laterally translated in steps of $210 \mathrm{~nm}$ (Figure 3d). At every position, Raman spectra were acquired. The wellknown Raman spectrum of polystyrene, which is characterised by aromatic ring vibrations ${ }^{36}$, appeared in both the SAR and UAR channels (Figure $3 \mathrm{e}$ and $3 \mathrm{f}$ ). However, the intensity of the SAR peaks decayed faster than that of the UAR peaks, and the SAR peaks were indistinguishable from the noise level at a distance of farther than $1260 \mathrm{~nm}$ from the centre of the sphere, $\sim 3 / 4$ of the sphere radius.

In classical spontaneous Raman scattering, the Raman intensity is proportional to the number of scattering centres contributing to the signal, which directly depends on the excited volume. Therefore, progressive intensity decay is expected when the objective is moved from the centre towards the edge of a sphere, because of the decrease in the excited volume. Taking advantage of the spherical geometry, we estimated the portion of the polymer sphere contained within the classical confocal collection volume as a spherical segment of volume $\pi h\left(3 a^{2}+3 b^{2}+h^{2}\right) / 6$, where $h$ is the thickness of the spherical segment, here defined by the size of the laser focal spot at the interface $(550 \mathrm{~nm})$, and $a$ and $b$ are the lower and higher radii of the segment. The corresponding spherical segment volume was thus calculated for every position from $X_{0}$ to $X_{6}$ (Figure $3 \mathrm{~d}$ ). For the last positions, $X_{7}$ and $X_{8}$, a spherical cap geometry was used, with $V=\pi h^{2}(3 a-h) / 3$. Despite its simplicity, this model provides a satisfactory description of the UAR signal decay (Figure 4a). The evolution of the UAR and SAR intensities with the axial distance between the sphere surface and the coverslip is displayed in Figure $4 \mathrm{~b}$. The experimental decay of the SAR signal was compared with the $z$-dependent supercritical decay ${ }^{37,38}$. Thus, we confirmed that the SAR intensity was strongly dominated by the axial distance between the microsphere surface and the refractive index discontinuity. This result was in contrast to the UAR signal intensity, which was primarily dependent on the number of scattering centres within the collection 
a
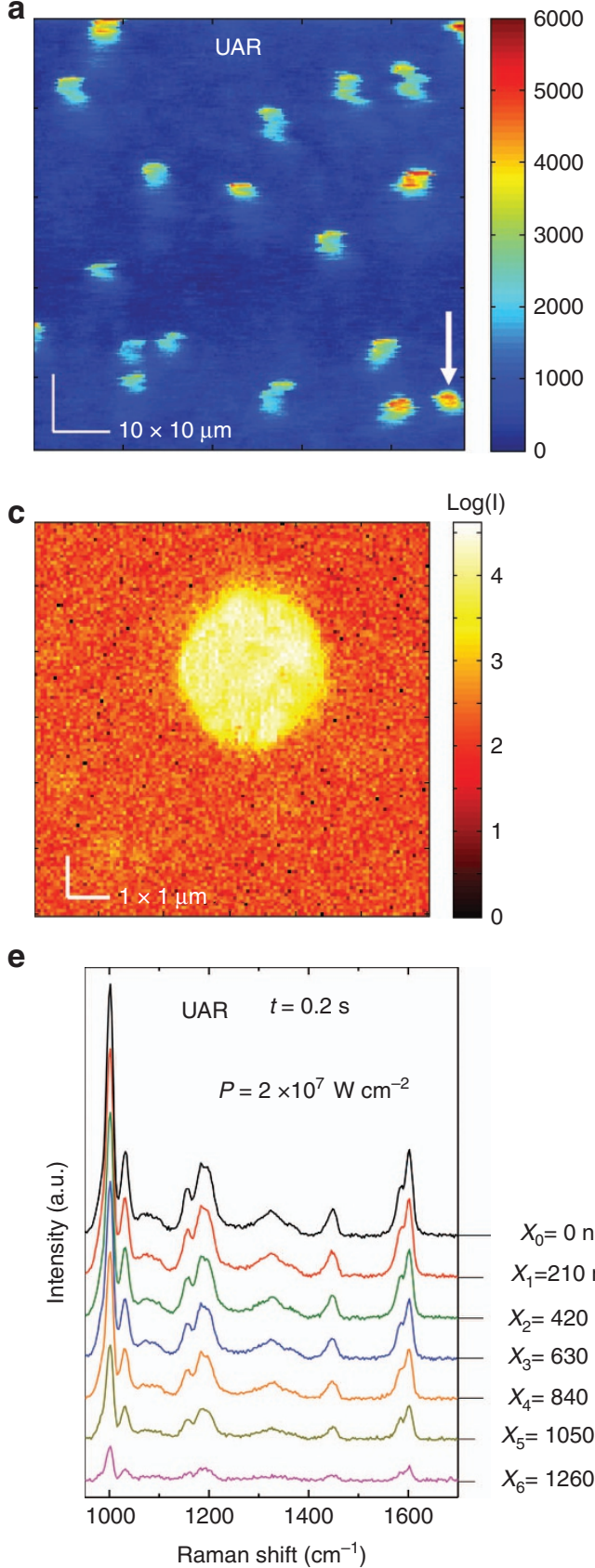

b

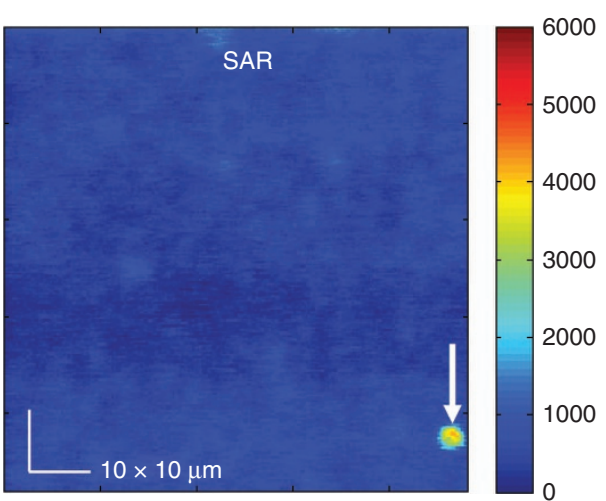

d

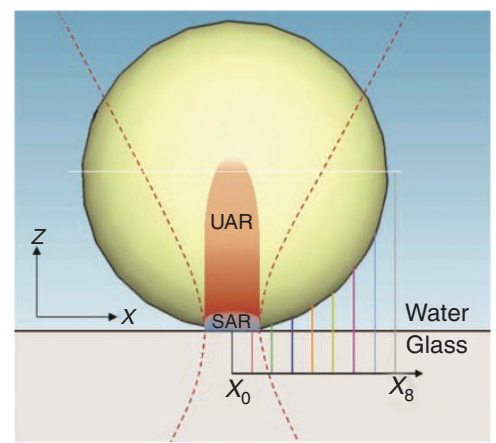

f

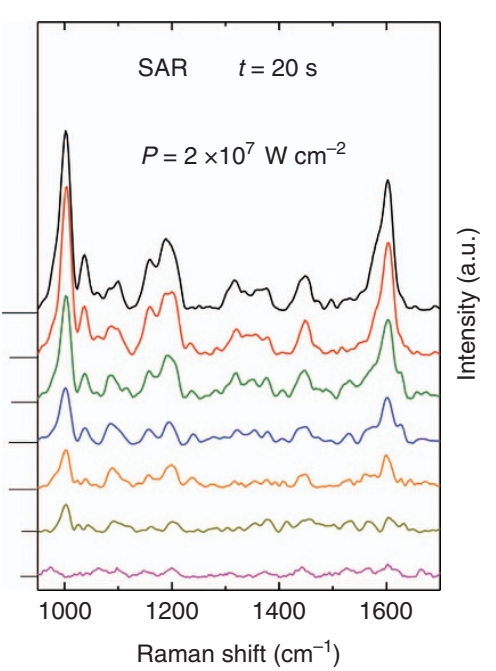

Figure 3 Simultaneous UAR and SAR imaging and spectroscopic analysis of a single polymer microsphere in water $\left(\lambda_{\mathrm{exc}}=633 \mathrm{~nm}\right)$. (a) UAR and (b) SAR imaging of an aqueous suspension of polystyrene microspheres. The images were obtained by selectively filtering the $1000 \mathrm{~cm}^{-1}$ polystyrene transition. (c) Microsphere with an average diameter of $3.3 \mu \mathrm{m}$ found immobilised on the coverslip surface. (d) Scheme depicting the lateral positions at which Raman spectra were acquired. The expected UAR and SAR collection volumes are represented at their corresponding scales ${ }^{35}$. (e) UAR and (f) SAR polystyrene spectra. The excitation power was set to $0.5 \mathrm{~mW}$ for imaging and to $50 \mathrm{~mW}$ for spectral acquisition, thus resulting in power densities on the order of $2 \times 10^{5}$ and $2 \times 10^{7} \mathrm{~W} \mathrm{~cm}^{-2}$, respectively. Exposure times of 0.2 and $20 \mathrm{~s}$ were chosen to ensure that at the starting point $X_{0}$, the polystyrene Raman modes would have comparable peak intensities in both the UAR and SAR collection channels.

volume (Figure 4a). Thus, the SAR sensitivity appeared to be limited to a few hundreds of nanometres in depth, with a detection efficiency decrease on the order of $80 \%$ after $200 \mathrm{~nm}$.

A closer look into the UAR and SAR spectra revealed noticeable differences (Figure 4c), the most remarkable being the relative intensity change between the 1000 and $1600 \mathrm{~cm}^{-1}$ modes. Variations in this particular ratio are often related to the length of the polymer chains or the degree of polymerisation ${ }^{39}$. A lower ratio does, in fact, correspond to a lower degree of polymerisation. In particular, the observed ratios of 2.9 in the UAR signal and 1.4 in the SAR signal may indicate a lower degree of polymerisation on the sphere surface, possibly because of polymer degradation mechanisms $\mathrm{s}^{40}$. In addition, some additional peaks were distinguished in the SAR spectrum, for instance, near $1380 \mathrm{~cm}^{-1}$. This vibration mode is most likely attributable to the known presence of functional carboxylate groups on the sphere surface ${ }^{41}$. Further investigations would be required to 
a

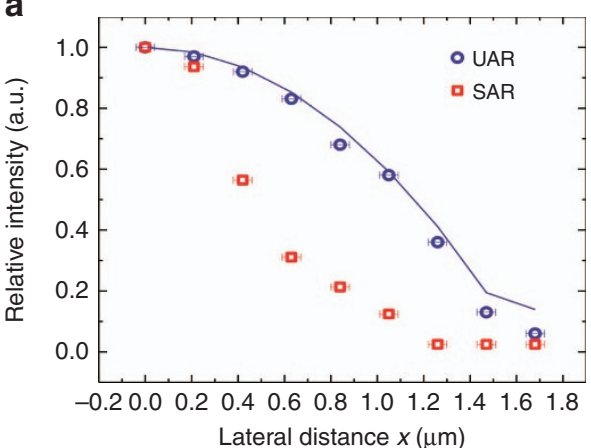

b

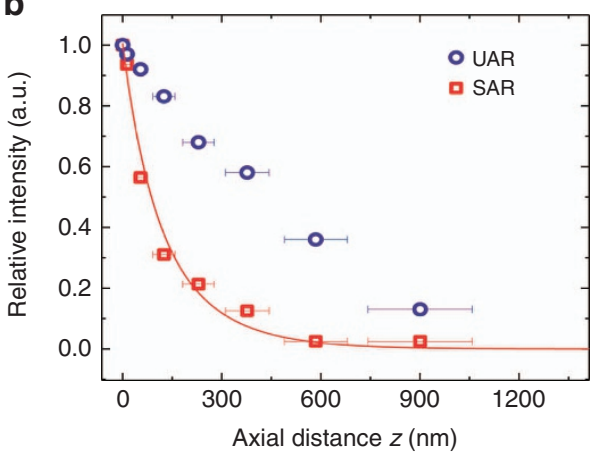

C

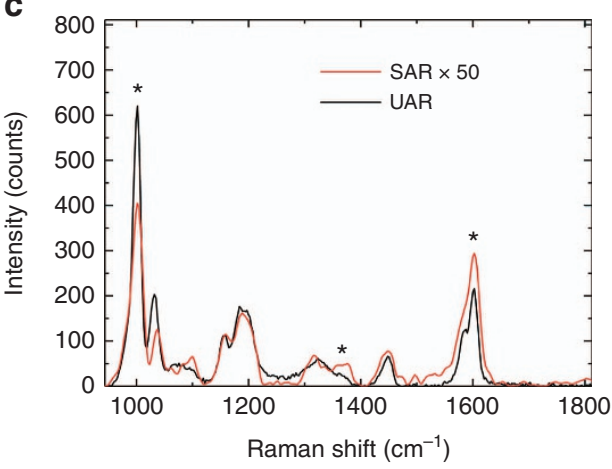

Figure 4 UAR and SAR intensity evolution and comparison of their spectral content. (a) Evolution of the $1000 \mathrm{~cm}^{-1}$ peak intensity as a function of lateral displacement. The solid line represents the progressive reduction in the excited volume determined from the spherical geometry. The error bars represent the experimental uncertainty associated with the lateral positions, estimated in steps of $\pm 40 \mathrm{~nm}$. (b) Evolution of the $1000 \mathrm{~cm}^{-1}$ peak intensity as a function of axial distance. The solid line represents the $z$-dependent decay of the supercritical emission for a radiation wavelength of $676 \mathrm{~nm}\left(1000 \mathrm{~cm}^{-1}\right.$ from excitation). The error bars show the uncertainty in $z$ derived from that in $x$ through simple geometric calculations. (c) Comparison of the UAR and SAR spectra acquired at the centre of the sphere $\left(X_{0}\right)$.

provide a conclusive explanation of the differences observed between the UAR and SAR spectra. Nevertheless, the SAR spectrum is clearly able to provide specific information about surfaces and boundaries, and to discriminate their contribution from that of the bulk. Furthermore, the possibility of simultaneously collecting far-field and near-field spectral information is a unique feature of this technique and one of its most important advantages. The richness of the spectroscopic information obtained through SAR microscopy was dramatically increased by the comparison, as seen in Figure 4c. Such comparisons are not possible either in TIR Raman spectroscopy or in SERS. In TIR microscopy, axial confinement is ensured by evanescent wave excitation, such that the penetration into the sample is typically limited to a few hundred nanometres. In SERS, the strong enhancement effect boosts the signal originating from surface specimens and masks any spontaneous contribution from the bulk. In addition, the spectral changes observed in SERS spectra with respect to the classical Raman spectra obtained from the same molecular specimen are difficult to interpret. Indeed, their origin is often attributed to the SERS substrate and the complex electromagnetic enhancement mechanism. In contrast, the classical far-field excitation used here allows the probing of micron-size volumes and the selective collection of reliable spectroscopic information from surface-related specimens (SAR) and their environment (UAR).

As a straightforward application, SAR was used to detect octadecyltrichlorosilane (OTS) self-assembled monolayers (SAMs) coated on glass coverslips (see the Supplementary Information for details about the coating procedure). OTS SAM (Figure 5a) have standard thicknesses of $2.5 \mathrm{~nm}$, varying slightly with the orientation of the hydrocarbon chains ${ }^{42,43}$. A non-amplified Raman scattering investigation of OTS SAMs is challenging because of the intrinsically weak Raman scattered signals arising from such small structures. It can even become impossible if the scattering in the monolayer's environment is not negligible. Indeed, most previous Raman studies of this system have been conducted with electromagnetic enhancement ${ }^{44}$. To the best of our knowledge, the spontaneous Raman spectrum of an OTS SAM has been reported only once ${ }^{45}$, with low spectral quality.

The OTS coating here was investigated on the basis of both the SAR and UAR signals under several environmental conditions, specifically, in an aqueous medium and embedded in ethanol (Figure 5). The Raman spectrum of a well-organized OTS SAM covalently bound to silica $^{46}$ is displayed in Figure $5 \mathrm{~b}-5 \mathrm{~d}$. In the classical UAR spectrum, the signature of the surrounding water, with its typical O-H vibration band in the $3000-3500 \mathrm{~cm}^{-1}$ region, was visible. However, the SAR spectrum, the water background was highly suppressed because of the SAR axial confinement. Although the OTS SAM spectrum was clearly recognisable in both the UAR and SAR spectra, close inspection revealed differences in the branching ratio of the vibration modes (Figure $5 \mathrm{c}$ and $5 \mathrm{~d}$ ), more precisely, between the symmetric and asymmetric stretching modes. The symmetric stretching modes $\left(\sim 1126 \mathrm{~cm}^{-1}\right.$ for $\mathrm{C}-\mathrm{C}$ and $\sim 2850 \mathrm{~cm}^{-1}$ for $\mathrm{CH}_{2}$ (Ref. 46)) appear weaker than the asymmetric modes in the SAR spectrum. This result cannot be explained by chemical or structural differences between the surface molecules and those in the bulk, because an OTS SAM is a 2D surface-bound structure. In other words, both the SAR and UAR signals collected here originated from the same molecules. Instead, this branching ratio change appeared to be due to a difference in the orientation of the induced electric dipole between the symmetric and asymmetric vibration modes. Spectral changes similar to those observed in Figure 5d have been reported in polarised vibrational spectroscopy. In particular, the symmetric $\mathrm{CH}_{2}$ stretching mode quenches in the so-called 'sp' and 'ps' spectra ${ }^{47}$, in which the emission polarisation is selected to be perpendicular to the excitation polarisation, thus indicating that the symmetric mode remains strictly parallel to the excitation polarisation, in contrast to the asymmetric mode. According to the simulations shown in Figure 2, quenching of the symmetric mode or boosting of the asymmetric mode can be expected for SAR collection, because perpendicular dipolar contributions are preferentially gathered in this case.

Water is a favourable medium for Raman investigations because it is a low-scattering medium and does not present strong peaks in the typical molecular fingerprint region $\left(800-1600 \mathrm{~cm}^{-1}\right)$. Therefore, 

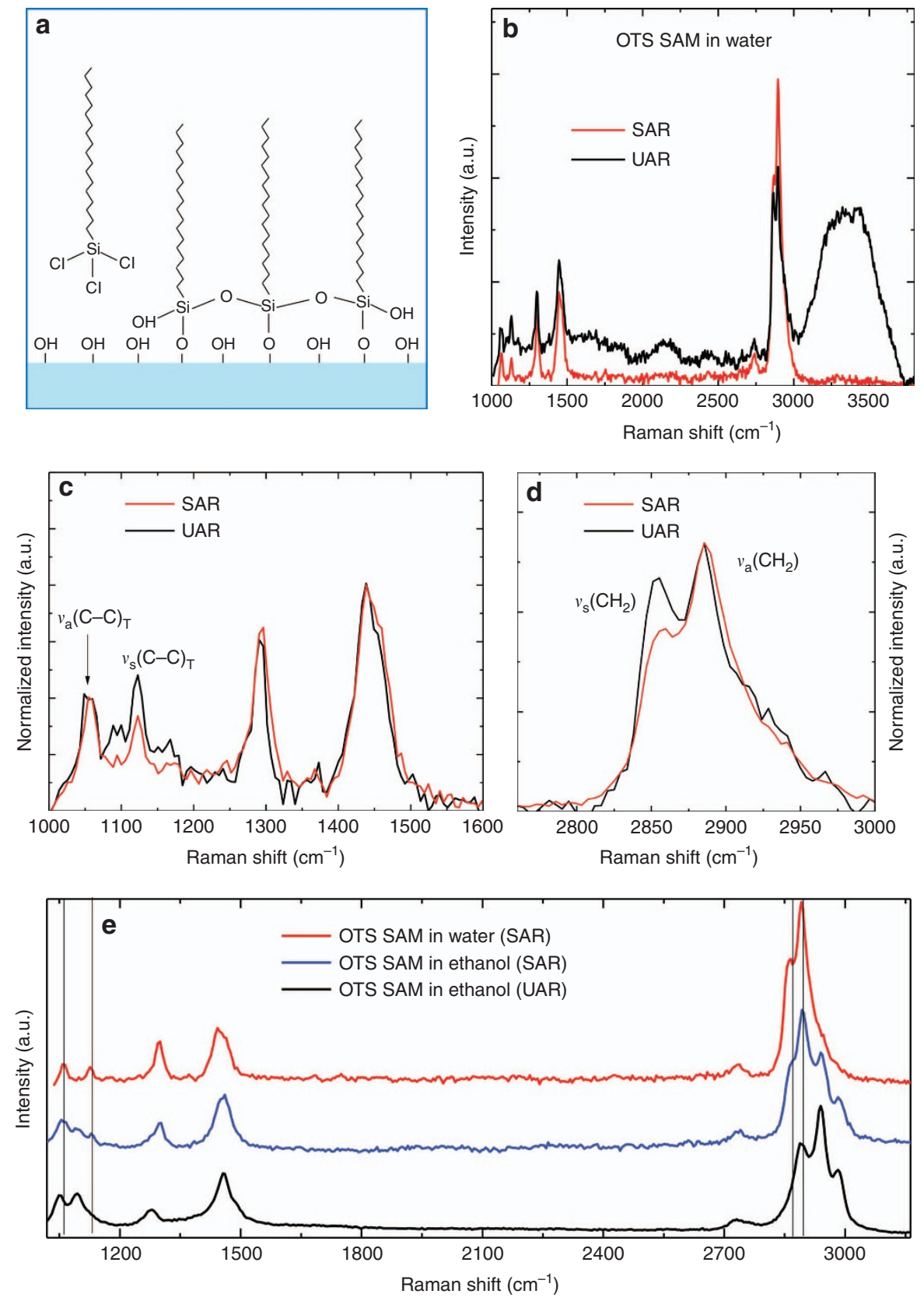

Figure 5 OTS SAM preparation and spectroscopic investigation. (a) Reaction leading to the formation of OTS SAMs. OTS molecules react with the $\mathrm{OH}$-activated surface, thus giving rise to siloxane ( $\mathrm{Si}-\mathrm{O}-\mathrm{Si}$ ) covalent bonds. (b) SAR and UAR measurements of an OTS-coated coverslip in an aqueous medium. (c) Comparison of the SAR and UAR OTS SAM spectra in the 1000-1600 $\mathrm{cm}^{-1}$ spectral region. (d) Comparison of the SAR and UAR OTS SAM spectra in the $2800-3000 \mathrm{~cm}^{-1}$ spectral region. (e) SAR and UAR spectra of the OTS-coated coverslip in ethanol. The vertical lines indicate the vibrations associated with the silane molecules. All measurements were performed with a power density of $2 \times 10^{7} \mathrm{~W} \mathrm{~cm}^{-2}$ and an exposure time of $10 \mathrm{~s}$.

using SAR collection instead of the classical confocal UAR collection approach was not critical for identifying the presence of the coating in this case. In contrast, as previously mentioned, Raman detection of an OTS SAM in an interfering environment is not trivial. To probe the SAR potential in such a case, we poured a generous amount of ethanol $(n=1.3616)$ over an OTS-coated coverslip. Ethanol molecules present a recognisable Raman spectrum with several strong peaks within the fingerprint region ${ }^{48}$. As seen from the UAR and SAR analysis, only the well-known vibrations of ethanol molecules were collected through the UAR channel (Figure 5e). Thus, a classical confocal investigation would not permit identification of the OTS monolayer under these experimental conditions. However, the presence of the OTS coating was clear in the SAR spectrum. The ethanol contribution was also noticeable in Figure 5e, but it was not sufficiently dominant to mask the Raman peaks associated with the surface-bound silane molecules that were previously identified in the water environment (Figure 5e).

Thus, we demonstrated in detail the potential of the SAR technique for performing surface-selective non-amplified Raman spectroscopy investigations. However, despite the promising performance of SAR collection, the inherently weak spontaneous Raman scattering mechanism can be difficult to exploit in investigations that prove to be particularly challenging, such as extremely low molecular 


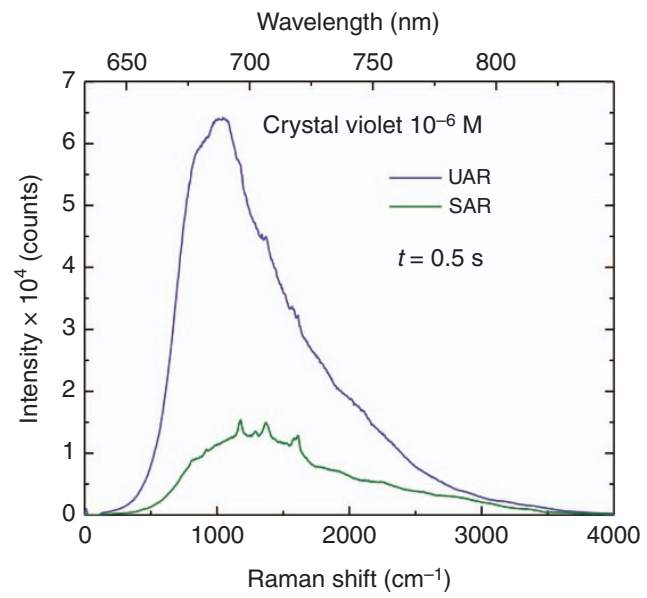

Figure 6 SAR detection of SERRS. Because of the resonant excitation of crystal violet at $633 \mathrm{~nm}$, both fluorescence emission and enhanced Raman scattering are produced and collected. The Raman signal from the coverslip surface is evident in the SAR spectrum, whereas the UAR spectrum is dominated by the fluorescence emission from the bulk solution.

concentrations and single molecules ${ }^{15}$. In such cases, SAR is not mutually exclusive but instead perfectly compatible with amplified Raman modalities. Therefore, the SAR detection of SERS is trivial and may be interesting for further limiting background contributions in SERS experiments. It is important to note that the detection threshold of such a SERS-SAR hybrid detection system will be limited by the enhancement factor achieved in the SERS hotspots. Therefore, it should be possible to reach the single-molecule level with an appropriate SERS substrate ${ }^{15}$. The only important requirement for a SERS substrate to be integrated into a SAR detection set-up is that it should exhibit a reasonable degree of transparency at the excitation wavelength and a suitable refractive index. Efficient transparent SERS substrates have been reported ${ }^{49}$; therefore, this should not constitute a limitation to the combination of the two techniques.

As an example, a solution of crystal violet dye $\left(\mathrm{C}_{25} \mathrm{H}_{30} \mathrm{ClN}_{3}\right)$ in water with a concentration of $10^{-6} \mathrm{M}$ was spectrally probed by using a gold-nanoparticle-activated coverslip (see the Supplementary Information). This dye is resonantly excited at $633 \mathrm{~nm}^{50}$, thus giving rise to fluorescence emission. In contrast, surface-enhanced resonance Raman scattering (SERRS) signals arise from molecules adsorbed on or in proximity to gold nanoparticles, because of surface plasmon resonance enhancement ${ }^{51}$. Thus, a competition is established between the fluorescence and Raman emissions. This competition was observed in the parallel SAR/UAR measurement presented in Figure 6. The amplified Raman peaks that are characteristic of crystal violet ${ }^{52}$ clearly appeared in the SAR spectrum. In contrast, the UAR spectrum was primarily dominated by the fluorescence signal coming from free crystal violet molecules in the solution. Once again, the axial selectivity of the SAR signal provided direct insight into the surface specimens with limited background interference, in this particular case, from the strong fluorescence background. Thus, the SAR detection of amplified Raman signals is experimentally straightforward and is advantageous for suppressing background contributions. Furthermore, with the proposed parallel near-field and far-field collection approach, it would be conceivable to perform investigations with dye-labelled molecules, thus enabling the analysis of both fluorescence features (e.g. molecular dynamics, through fluorescence correlation spectroscopy ${ }^{53}$ ) and Raman fingerprints. It is also notable that in its current state, our experimental set-up might be used as a simultaneous Raman- fluorescence microscope. Raman scattering and fluorescence emission are spectroscopic methods that are difficult to combine in a single experiment. Here, this incompatibility was overcome by taking advantage of the availability of two well-distinguished collection volumes, one with a near-field extent and one with the classical farfield extent.

\section{CONCLUSION}

In this work, we presented a new Raman modality, namely, SAR microscopy, and demonstrated its use in performing surface-selective non-amplified Raman scattering investigations of nanoscale specimens with an improved signal-to-background ratio. The technique was applied for the detection of OTS SAMs, and was shown to be compatible with other Raman modalities such as SERRS.

\section{CONFLICT OF INTEREST}

The authors declare no conflict of interest.

\section{ACKNOWLEDGEMENTS}

This work was supported by the Swiss National Science Foundation (SNF). We would like to thank Dorinel Verdes for advice throughout the execution of this project.

1 Kneipp K, Kneipp H, Itzkan I, Dasari RR, Feld MS. Ultrasensitive chemical analysis by Raman spectroscopy. Chem Rev 1999; 99: 2957-2976.

2 Pupples GJ, de Mul FFM, Otto C, Greve J, Robert-Nicoud M et al. Studying single living cells and chromosomes by confocal Raman microspectroscopy. Nature 1990; 347: 301-303.

3 Eckmann A, Felten A, Mishchenko A, Britnell L, Krupke R et al. Probing the nature of defects in graphene by Raman spectroscopy. Nano Lett 2012; 12: 3925-3930.

4 Penney CM, Goldman LM, Lapp M. Raman scattering cross sections. Nat Phys Sci 1972; 235: 110-112.

5 Schuster KC, Reese I, Urlaub E, Gapes JR, Lendl B. Multidimensional information on the chemical composition of single bacterial cells by confocal Raman microspectroscopy. Anal Chem 2000; 72: 5529-5534.

6 Webb RH. Confocal optical microscopy. Rep Prog Phys 1996; 59: 427-471.

7 Everall NJ. Confocal Raman microscopy: why the depth resolution and spatial accuracy can be much worse than you think. App/ Spectrosc 2000; 54: 1515-1520.

8 Eesley GL Coherent Raman Spectroscopy. Oxford: Pergamon Press; 1981.

9 Campion A, Kambhampati P. Surface-enhanced Raman scattering. Chem Soc Rev 1998; 27: 241-250.

10 Fast A, Kenison JP, Syme CD, Potma EO. Surface-enhanced coherent anti-Stokes Raman imaging of lipids. App/ Opt 2016; 55: 5994-6000.

11 Giergiel J, Reed CE, Hemminger JC, Ushioda S. Surface plasmon polariton enhancement of Raman scattering in a Kretschmann geometry. J Phys Chem 1988; 92 5357-5365.

12 Camp Jr CH, Cicerone MT. Chemically sensitive bioimaging with coherent Raman scattering. Nat Photonics 2015; 9: 295-305.

13 Li JF, Huang YF, Ding Y, Yang ZL, Li SB et al. Shell-isolated nanoparticle-enhanced Raman spectroscopy. Nature 2010; 464: 392-395.

14 Freudiger CW, Min W, Saar BG, Lu SJ, Holtom GR et al. Label-free biomedical imaging with high sensitivity by stimulated Raman scattering microscopy. Science 2008; 322 : 1857-1861.

15 Kneipp K, Wang Y, Kneipp H, Perelman LT, Itzkan I et al. Single molecule detection using surface-enhanced Raman scattering (SERS). Phys Rev Lett 1997; 78: 1667-1670.

16 Pestov D, Wang X. Ariunbold GO, Murawski RK, Sautenkov VA et al. Single-shot detection of bacterial endospores via coherent Raman spectroscopy. Proc Natl Acad Sci USA 2008; 205: 422-427.

17 Dudovich N, Oron D, Silberberg Y. Single-pulse coherently controlled nonlinear Raman spectroscopy and microscopy. Nature 2002; 418: 512-514.

18 Lin XM, Cui Y, Xu YH, Ren B, Tian ZQ. Surface-enhanced Raman spectroscopy: substrate-related issues. Anal Bioanal Chem 2009; 394: 1729-1745.

19 Tantra R, Brown RJC, Milton MJT. Strategy to improve the reproducibility of colloidal SERS. J Raman Spectrosc 2007; 38: 1469-1479.

20 Xiu ZM, Zhang QB, Puppala HL, Colvin VL, Alvarez PJJ. Negligible particle-specific antibacterial activity of silver nanoparticles. Nano Lett 2012; 12: 4271-4275.

21 Saar BG, Freudiger CW, Reichman J, Stanley CM, Holtom GR et al. Video-rate molecular imaging in vivo with stimulated Raman scattering. Science 2010; 330: $1368-1370$. 
22 Potma EO, de Boeij WP, van Haastert PJM, Wiersma DA. Real-time visualization of intracellular hydrodynamics in single living cells. Proc Natl Acad Sci USA 2001; 98: 1577-1582.

23 Zumbusch A, Holtom GR, Xie XS. Three-dimensional vibrational imaging by coherent anti-stokes Raman scattering. Phys Rev Lett 1999; 82: 4142-4145.

24 Fu Y, Wang HF, Shi RY, Cheng JX. Characterization of photodamage in coherent antiStokes Raman scattering microscopy. Opt Express 2006; 14: 3942-3951.

25 Watanabe K, Palonpon AF, Smith NI, Chiu LD, Kasai A et al. Structured line illumination Raman microscopy. Nat Commun 2015; 6: 10095.

26 Iwamoto R, Miya M, Ohta K, Mima S. Total internal reflection Raman spectroscopy as a new tool for surface analysis. J Am Chem Soc 1980; 102: 1212-1213.

27 Woods DA, Bain CD. Total internal reflection Raman spectroscopy. Analyst 2012; 137: $35-48$.

28 Lukosz W, Kunz RE. Light emission by magnetic and electric dipoles close to a plane dielectric interface. I. Total radiated power. J Opt Soc Am 1977; 67: 1607-1615.

29 Lukosz W. Light emission by magnetic and electric dipoles close to a plane dielectric interface. III. Radiation patterns of dipoles with arbitrary orientation. J Opt Soc Am 1979; 69: 1495-1503.

30 Novotny L. Allowed and forbidden light in near-field optics. I. A single dipolar light source. J Opt Soc Am A 1997; 14: 91-104.

31 Winterflood CM, Ruckstuhl T, Verdes D, Seeger S. Nanometer axial resolution by threedimensional supercritical angle fluorescence microscopy. Phys Rev Lett 2010; 105: 108103.

32 Bourg N, Mayet C, Dupuis G, Barroca T, Bon P et al. Direct optical nanoscopy with axially localized detection. Nat Photonics 2015; 9: 587-593.

33 Rabe M, Soragni A, Reynolds NP, Verdes D, Liverani E et al. On-surface aggregation of $\alpha$-Synuclein at nanomolar concentrations results in two distinct growth mechanisms. ACS Chem Neurosci 2013; 4: 408-417.

34 Mout R, Moyano DF, Rana S, Rotello VM. Surface functionalization of nanoparticles for nanomedicine. Chem Soc Rev 2012; 41: 2539-2544.

35 Ruckstuhl T, Verdes D, Winterflood CM, Seeger S. Simultaneous near-field and far-field fluorescence microscopy of single molecules. Opt Express 2011; 19: 6836-6844.

36 Anema JR, Brolo AG, Felten A, Bittencourt C. Surface-enhanced Raman scattering from polystyrene on gold clusters. J Raman Spectrosc 2010; 41: 745-751.

37 Ruckstuhl T, Rankl M, Seeger S. Highly sensitive biosensing using a supercritical angle fluorescence (SAF) instrument. Biosens Bioelectron 2003; 18: 1193-1199.

38 Enderlein J, Ruckstuhl T, Seeger S. Highly efficient optical detection of surfacegenerated fluorescence. Appl Opt 1999; 38: 724-732.

39 Sears WM, Hunt JL, Stevens JR. Raman scattering from polymerizing styrene. I. Vibrational mode analysis. J Chem Phys 1981; 75: 1589-1598.

40 Guaita M, Chiantore O, Costa L. Changes in degree of polymerization in the thermal degradation of polystyrene. Polymer Degrad Stabil 1985; 12: 315-332.
41 Suh JS, Kim J. Three distinct geometries of surface-adsorbed carboxylate groups. J Raman Spectrosc 1998; 29: 143-148.

42 Wang YL, Lieberman M. Growth of Ultrasmooth octadecyltrichlorosilane self-assembled monolayers on $\mathrm{SiO}_{2}$. Langmuir 2003; 19: 1159-1167.

43 McGovern ME, Krishna MRK, Thompson M. Role of solvent on the silanization of glass with octadecyltrichlorosilane. Langmuir 1994; 10: 3607-3614.

44 Thompson WR, Pemberton JE. Raman spectroscopy of covalently bonded alkylsilane layers on thin silica films immobilized on silver substrates. Anal Chem 1994; 66: 3362-3370.

45 Bryant MA, Pemberton JE. Surface Raman scattering of self-assembled monolayers formed from 1-alkanethiols: behavior of films at Au and comparison to films at Ag. J Am Chem Soc 1991; 113: 8284-8293.

46 Calkins JA, Peacock AC, Sazio PJA, Allara DL, Badding JV. Spontaneous waveguide Raman spectroscopy of self-assembled monolayers in silica micropores. Langmuir 2011; 27: 630-636.

47 Itoh Y, Hasegawa T. Polarization dependence of Raman scattering from a thin film involving optical anisotropy theorized for molecular orientation analysis. J Phys Chem A 2012; 116: 5560-5570.

48 Mammone JF, Sharma SK, Nicol M. Raman spectra of methanol and ethanol at pressures up to 100 kbar. J Phys Chem 1980; 84: 3130-3134.

49 Wang HH, Cheng TY, Sharma P, Chiang FY, Chiu SWY et al. Transparent Ramanenhancing substrates for microbiological monitoring and in situ pollutant detection. Nanotechnology 2011; 22: 385702.

50 Lewis GN, Magel TT, Lipkin D. Isomers of crystal violet ion. Their absorption and reemission of light. J Am Chem Soc 1942; 64: 1774-1782.

51 Meyer SA, Le Ru EC, Etchegoin PG. Quantifying resonant Raman cross sections with SERS. J Chem Phys A 2010; 114: 5515-5519.

52 Kleinman SL, Ringe E, Valley N, Wustholz KL, Phillips E et al. Single-molecule surfaceenhanced Raman spectroscopy of crystal violet isotopologues: theory and experiment. J Am Chem Soc 2011; 133: 4115-4122.

53 Winterflood CM, Seeger S. Simultaneous surface-near and solution fluorescence correlation spectroscopy. J Fluoresc 2011; 26: 753-756.

(c) (i) (2) This work is licensed under a Creative Commons Attributionc. ${ }_{\mathrm{BY}} \mathrm{NC}$ SA NonCommercial-ShareAlike 4.0 International License. The images or other third party material in this article are included in the article's Creative Commons license, unless indicated otherwise in the credit line; if the material is not included under the Creative Commons license, users will need to obtain permission from the license holder to reproduce the material. To view a copy of this license, visit http:// creativecommons.org/licenses/by-nc-sa/4.0/

(C) The Author(s) 2017

Supplementary Information for this article can be found on the Light: Science \& Applications' website (http://www.nature.com/lsa). 